was developed, which showed excellent targeting of the folate receptor beta on activated macrophages in synovial tissue in a preclinical arthritic rat model (3). Objectives: To assess the value of $\left[{ }^{18} \mathrm{~F}\right] \mathrm{fluoro}-\mathrm{PEG}$-folate PET-CT for imaging inflamed joints in patients with clinically active RA.

Methods: Nine RA patients with at least two clinically inflamed hand joints were included. PET-CT scans of the hands were acquired after intravenous administration of either $185 \mathrm{MBq}$ of $\left[{ }^{18} \mathrm{~F}\right]$ fluoro-PEG-folate $(\mathrm{n}=6)$ or $425 \mathrm{MBq}$ of $(R)-\left[{ }^{11} \mathrm{C}\right] \mathrm{PK} 11195(\mathrm{n}=3)$. Volumes of Interest (VOI) were drawn over joints with visually marked uptake and Standardized Uptake Values (SUVs) were calculated. Background VOls were drawn on metacarpal bone in order to calculate Target-to-Background (T/B) ratios.

Results: No side effects were observed, establishing the safety of $\left[{ }^{18} \mathrm{~F}\right]$ fluoroPEG-folate for use in humans. $\left[{ }^{18} \mathrm{~F}\right]$ fluoro-PEG-folate clearly showed uptake in arthritic joints, as shown in Figure 1. In patients scanned with $\left[{ }^{18} \mathrm{~F}\right]$ fluoro-PEGfolate, 25 positive joints were seen, with a minimum of two joints per patient. Clinical arthritis was confirmed in 10 of these 25 joints, and was absent in 15 positive joints, suggesting the presence of subclinical inflammation. Whilst both $\left[{ }^{18} \mathrm{~F}\right]$ fluoro-PEG-folate and $\left.(R)-{ }^{11} \mathrm{C}\right] \mathrm{PK} 11195$ accumulated in arthritic joints, $\left[{ }^{18} \mathrm{~F}\right]$ fluoro-PEG-folate showed a significantly lower background uptake than $(R)-\left[{ }^{11} \mathrm{C}\right] \mathrm{PK} 11195$ (SUV of 0.18 vs $0.75 ; p<0.001$ ) respectively. T/B-ratios were significantly higher for $\left[{ }^{18} \mathrm{~F}\right]$ fluoro-PEG-folate (3.60vs $1.72, \mathrm{p}=0.009$ ).

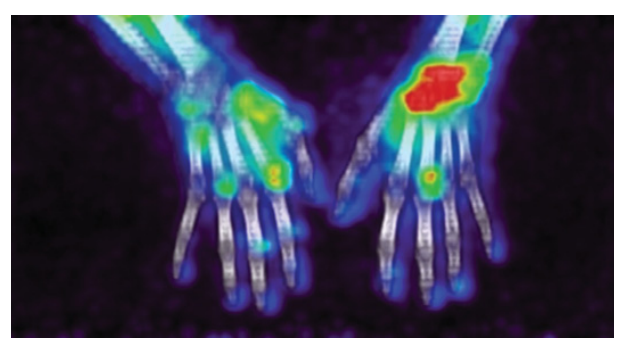

Conclusions: This first in patient study clearly demonstrates the potential of $\left[{ }^{18} \mathrm{~F}\right]$ fluoro-PEG-folate PET-CT as macrophage tracer to image both clinically and sub-clinically affected joints in RA patients. $\left[{ }^{18} \mathrm{~F}\right]$ fluoro-PEG-folate showed better characteristics for arthritis imaging than the established tracer $(R)-\left[{ }^{11} \mathrm{C}\right] \mathrm{PK} 11195$ because of its lower background signal.

References:

[1] Gent YY, et al. J Rheumatology. 2014; 41: 2145-52.

[2] Gent YY, et al. Arthritis Rheum. 2012; 64: 62-6.

[3] Gent YY, et al. Arthritis Res Ther. 2013; 15: R37.

Disclosure of Interest: None declared

DOI: 10.1136/annrheumdis-2017-eular.3249

\section{SAT0651 QUANTIFICATION OF DYNAMIC MRI EXAMINATIONS IN JUVENILE IDIOPATHIC ARTHRITIS}

N. Tzaribachev ${ }^{1}$, R. Hagoug $^{2}$, P. Louka ${ }^{2}$, J. Islam ${ }^{2}$, M. Hinton ${ }^{2}$, O. Kubassova ${ }^{2}$ M. Boesen ${ }^{3}{ }^{1}$ Pri - Pediatric Rheumatology Research Institute, Bad Bramstedt, Germany; ${ }^{2}$ Image Analysis Group, London, United Kingdom; ${ }^{3}$ Radiology, Copenhagen University Hospital Bispebjerg and Frederiksberg, Bispebjerg, Denmark

Background: In chronic inflammatory conditions, the need for a more objective measurement of disease activity has been identified. Imaging biomarkers as outcome measurements based on the automated quantification of dynamic contrast enhanced magnetic resonance images (DCE-MRI) have been studied in adult patients with rheumatoid arthritis (RA) ${ }^{1}$. In children with juvenile idiopathic arthritis (JIA) similar knowledge is very limited.

Objectives: To compare treatment related changes of clinical scores in patients with JIA and automated DCE-MRI quantitative parameters analyzed with a dedicated software Dynamika ${ }^{\text {tm }}$ also compared to clinical outcomes of the patients. Methods: In patients with polyarticular JIA with insufficient ( $\geq 3$ affected joints) response or intolerance to $\geq 3$ months Methotrexate, Etanercept was started. Six Slice Axial DCE-MRI of the metacarpophaleangeal (MCP) 2-5 joints in the clinically most affected hand was performed at 3 time points: baseline (BL), month 3 and 6 of treatment using a 0.2 Tesla Esaote C-Scan. Clinical scores included active joint (AJ) counts. Clinical response was considered a state of $\leq 3$ AJ. DCE-MRI was analyzed using regions of interest (ROI) covering synovium in slices where MCPs $2-5$ were visible. Output parameters included dynamic MRI quantification scores (DEMRIQvol) corresponding to the volume of enhancing voxels within the synovial ROIs alone or multiplied with the mean of the maximum enhancement (ME) or the initial rate of enhancement (IRE). Differences in DEMRIQvol scores between visits were analyzed using t-test $\left(p<0.05^{*}=\right.$ statistically significant, $p<0.25^{\star \star}=$ clinically meaningful). Concordance between clinical and DEMRIQvol scores were described.

Results: 18 Caucasian patients (12 girls, median age 12,6 years, median disease duration 1,2 years) were included in the study. Two patients discontinued imaging after BL but continued treatment. In all but 3 of the remaining patients statistically significant and/or clinically meaningful changes were documented for DEMRIQvol ME between visits.
In 4 patients clinical and DEMRIQvol scores showed corresponded changes but these were non-concordant in all others patients.

Based on DEMRIQvol change (irrespective of the clinical scores) the outcome of the patient could be predicted:

- in 5 patients improvement of DEMRIQvol scores predicted response to treatment (within 2-6 months after last MRI examination)

- in 4 patients the increase or the persistence of a high DEMRIQvol predicted non-response to treatment

- in 7 patients increase in DEMRIQvol (after initial decrease) or persistence of a high DEMRIQ vol predicted flare (in 3 of the patients flare occurred after treatment discontinuation)

In all patients subclinical disease could be detected on MRI in clinically unaffected joints.

Conclusions: Dynamika based scores appear to be useful for depicting disease activity in JIA and seem to support clinical examination by detecting subclinical inflammation. More over, in the present study DEMRIQvol scores were predictive for the outcome of the patients and were able to "foresee" response to treatment, flare of disease, non-response to treatment in most patients possibly making DEMRIQvol scores supportive in research and clinical decision taking.

References:

[1] Kubassova O et al; Eur J Radiol. 2010 Jun;74(3):e67-72.

Acknowledgements: The study was supported by Pfizer.

Disclosure of Interest: N. Tzaribachev: None declared, R. Hagoug Employee of: Image Analysis Group, P. Louka Employee of: Image Analysis Group, J. Islam Employee of: Image Analysis Group, M. Hinton Employee of: Image Analysis Group, O. Kubassova Employee of: Image Analysis Group, M. Boesen Shareholder of: Image Analysis Group

DOI: 10.1136/annrheumdis-2017-eular.4341

\section{SAT0652 CLINICAL AND IMMUNOLOGICAL SIGNIFICANCE OF RADIOGRAPHIC THYMIC ALTERATIONS IN PATIENTS WITH RHEUMATOID ARTHRITIS}

O. Murata ${ }^{1}$, K. Suzuki $^{1}$, H. Sugiura ${ }^{2}$, Y. Kondo ${ }^{1}$, M. Takeshita ${ }^{1}$, H. Yasuoka ${ }^{1}$ K. Yamaoka ${ }^{1}, K_{\text {Koga }}{ }^{3}$, R. Morita ${ }^{4}$, A. Yoshimura ${ }^{4}$, T. Takeuchi ${ }^{1} .{ }^{1}$ Division of Rheumatology, Department of Internal Medicine, School of Medicine; ${ }^{2}$ Division of Diagnostic Radiology, Department of Radiology, School of Medicine, Keio University, Tokyo; ${ }^{3}$ Inflammation Drug Discovery Unit, Pharmaceutical Research Division, Takeda Pharmaceutical Company Limited, Kanagawa: ${ }^{4}$ Department of Microbiology and Immunology, School of Medicine, Keio University, Tokyo, Japan

Background: The thymus, a primary lymphoid organ, plays a crucial role in immune system homeostasis $[1,2]$. Although several small-scale studies of the association between radiographic thymus alterations and serological features have been reported in systemic autoimmune diseases, information in patients with rheumatoid arthritis (RA) is limited.

Objectives: We conducted a large-scale cross-sectional analysis of radiographic thymus alterations and their association with clinical and immunological features in patients with RA.

Methods: RA patients were randomly selected from all patients who visited our department and underwent chest CT scan between January 2013 and December 2015. Patients with thymoma or thymic cyst and those aged less than 30 years were excluded. Thymic enlargement and thymus attenuation score in axial images of CT scans were quantitatively interpreted. We defined thymic enlargement as a thickness of more than $13 \mathrm{~mm}$ and graded the score by a four-point scale (score $0-3)$ according to previous studies $[3,4]$. Associations with immunophenotyping data of peripheral blood by flow cytometry and clinical and serological information were statistically analyzed in some available patients.

Results: 387 RA patients were enrolled. $78 \%$ were women and mean age was $65.2 \pm 12.3$ years. Thymic enlargement was found in $76(19.6 \%)$ patients. Thymus attenuation (score $>2$ ) was found in $154(39.8 \%)$ patients. These findings were more frequent than in undiagnosed controls $(11.3 \%(P=0.078)$ and $22.5 \%$ $(P=0.017))$. Importantly, radiographic thymus alterations in these RA patients, especially thymus attenuation score, were significantly associated with serological features such as serum level of CRP, ESR, IgG, RF-positivity or ACPA-positivity $(P=0.0003,0.001,0.0009,0.005$, and 0.0009 respectively). When we investigated the association with the proportion of 68 peripheral blood subpopulations in 83 RA patients, thymic enlargement was significantly associated with the proportions of CD45RA+CCR7+ naïve CD4+T cell or CD45RO+CCR7-effector memory $\mathrm{CD} 4+\mathrm{T}$ cell $(P=0.04$ and 0.009$)$. Furthermore, thymus attenuation score was also significantly associated with the proportions of $\mathrm{CD} 4+$ naĩve T cell, CD4+effector memory T cell, CXCR5-CXCR3+CXCR6+CD4+Th1/Th17 cell, CD45RO-CCR7+CD95+CD4+ stem cell memory T cell, and CD19+ B cell $(P=0.03,0.02,0.04,0.04$, and 0.037 respectively).

Conclusions: Radiographic thymus alterations are frequent in RA patients and may reflect immunological features of autoantibody production or $\mathrm{T}$ cell differentiation and function.

References:

[1] Gorozny JJ, et al. Trends Immunol 2001;22:251-255.

[2] Seddon B, et al. Immunol Today 2000;21:94-99.

[3] Naidich P, et al. Lippincott-Raven 1999:57-73.

[4] Ackman JB, et al. Radiology 2013;268(1):245-253.

Disclosure of Interest: None declared 